\title{
The extended Baryon Oscillation Spectroscopic Survey (eBOSS): testing a new approach to measure the evolution of the structure growth
}

\author{
Rossana Ruggeri ${ }^{1 \star}$, Will J. Percival ${ }^{1}$, Eva-Maria Mueller ${ }^{1}$, Héctor Gil-Marín ${ }^{2,3}$, \\ Fangzhou Zhu ${ }^{4}$, Nikhil Padmanabhan ${ }^{4}$, Gong-Bo Zhao ${ }^{5,1}$ \\ 1 Institute of Cosmology 8 Gravitation, University of Portsmouth, Dennis Sciama Building, Portsmouth, PO1 3FX, UK \\ 2 Sorbonne Université, Institut Lagrange de Paris (ILP), 98 bis Boulevard Arago, 75014 Paris, France \\ 3 Laboratoire de Physique Nucléaire et de Hautes Energies, Université Pierre et Marie Curie, Paris, France \\ 4 Dept. of Physics, Yale University, New Haven, CT 06511 \\ 5 National Astronomy Observatories, Chinese Academy of Science, Beijing, 100012, P.R.China
}

17 September 2018

\begin{abstract}
The extended Baryon Oscillation Spectroscopic Survey (eBOSS) is one of the first of a new generation of galaxy redshift surveys that will cover a large range in redshift with sufficient resolution to measure the baryon acoustic oscillations (BAO) signal. For surveys covering a large redshift range we can no longer ignore cosmological evolution, meaning that either the redshift shells analysed have to be significantly narrower than the survey, or we have to allow for the averaging over evolving quantities. Both of these have the potential to remove signal: analysing small volumes increases the size of the Fourier window function, reducing the large-scale information, while averaging over evolving quantities can, if not performed carefully, remove differential information. It will be important to measure cosmological evolution from these surveys to explore and discriminate between models. We apply a method to optimally extract this differential information to mock catalogues designed to mimic the eBOSS quasar sample. By applying a set of weights to extract redshift space distortion measurements as a function of redshift, we demonstrate an analysis that does not invoke the problems discussed above. We show that our estimator gives unbiased constraints.
\end{abstract}

Key words: eBOSS, large-scale structure of Universe, dark energy, modified gravity, cosmology: observations.

\section{INTRODUCTION}

The eBOSS survey (Dawson et al. 2016, Zhao et al. 2016 Blanton et al. 2017), which commenced in July 2014, will cover the largest volume to date of any cosmological redshift survey with a density sufficient to extract useful cosmological information. eBOSS observations will target multiple density-field tracers, including more than 250, 000 luminous red galaxies (LRGs), 195, 000 emission line galaxies (ELGs) at effective redshifts $z=0.72,0.87$ and over 500,000 quasars between $0.8<z<2.2$. The survey's goals include the distance measurement at $1-2 \%$ accuracy with the BAO peak on the LRG sample and the first BAO measurements using quasars as density tracers over the redshift range $1<z<2$ (the first clustering measurements were recently presented

\footnotetext{
^ Email: rossana.ruggeri@port.ac.uk
}

in Ata et al. (2017)). The wide redshift range covered, compared with that in previous redshift surveys represents an unique opportunity to test and discriminate between different cosmological scenarios on the basis of their evolution in redshift. Full survey details can be found in Dawson et al. (2016).

The clustering analysis strategy adopted for most recent galaxy survey analyses was based on computing the correlation function or the power spectrum for individual samples or subsamples, overwhich the parameters being measured were assumed to be unvarying with redshift. The measurements were then considered to have been made at an effective redshift: see e.g. Alam et al. (2016), Anderson et al. (2014). In particular Alam et al. (2016), divided the full The Baryon Oscillation Spectroscopic Survey (BOSS) survey volume in three overlapping redshift bins and repeated the measurement in each sub-volume. This technique has

(C) $0000 \mathrm{RAS}$ 
many disadvantages: the choice of bins is a balance between having enough data for a significant detection in each bin leading to Gaussian errors and having bins small enough that there is no cosmological evolution across them, leading to a degrading compromise. The technique also ignores the correlation between galaxies in different redshift bins leading to a lower signal to noise ratio, which in Fourier space is equivalent to having a wider window function.

To complicate analyses further, many mock catalogues currently used to compare to the data intrinsically lack evolution, or "lightcone" effects, being drawn from simulation snapshots. Although this is a separate problem, these differences limit the tests of the effects of evolution that can be performed, and have the potential to hide biases caused by evolution.

Recent work by Zhu et al. (2015), Ruggeri et al. (2017), and Mueller et al. (2017) introduced an alternative approach to the redshift binning. The idea is to consider the whole volume of the survey and optimally compress the information in the redshift direction by applying a set of redshift weights to all galaxies, and only then computing the weighted correlation function. Comparing measurements made using different sets of redshift weights maintains the sensitivity to the underlying evolving theory. The sets of weights are derived in order to minimize the error on the parameters of interest. In addition, by applying the redshift weighting technique instead of splitting the survey, is it possible to compute the correlation function to larger scales whilst accounting for the evolution in redshift; this was particularly clear in Mueller et al. (2017), which considered this method to optimize the measurement of local primordial non gaussianity, which relies on large scales. Further, Zhu et al. (2016) showed that the application of a weighting scheme rather than splitting into bins also improves BAO measurements.

The need to correctly deal with evolution will increase for the DESI and Euclid experiments, which will cover a broad redshift range and have significantly reduced statistical measurement errors compared to current surveys in any particular redshift range. The Dark Energy Spectroscopic Instrument (DESI) ${ }^{1}$ is a new MOS currently under construction for the 4-meter Mayall telescope on Kitt Peak. DESI will be able to obtain 5000 simultaneous spectra, which coupled with the increased collecting area of the telescope compared with the 2.5-meter Sloan telescope, means that it can create a spectroscopic survey of galaxies $\sim 20$ times more quickly than eBOSS. In 2020 the European Space Agency will launch the Euclid 2 satellite mission. Euclid is an ESA medium class astronomy and astrophysics space mission, and will undertake a galaxy redshift survey over the redshift range $0.9<z<1.8$, while simultaneously performing an imaging survey in both visible and near-infrared bands. The complete survey will provide hundreds of thousands images and several tens of Petabytes of data. About 10 billion sources will be observed by Euclid out of which several tens of million galaxy redshifts will be measured and used to make galaxy clustering measurements.

In the current work we test the redshift weighting approach by analysing a set of 1000 mocks catalogues Chuang

1 http://desi.lbl.gov/ 2 http://sci.esa.int/euclid et al. 2015) designed to match the eBOSS quasar sample. This quasar sample has a low density $\left(82.6\right.$ objects $\left./ \mathrm{deg}^{2}\right)$ compared to that of recent galaxy samples, and covers a total area over $7500 \mathrm{deg}^{2}$. The quasars are highly biased targets and we expect their bias to evolve with redshift, $b(z) \propto c_{1}+c_{2}(1+z)^{2}$, with constant values $c_{1}=0.607 \pm 0.257$, $c_{2}=0.274 \pm 0.035$, as measured in Laurent et al. (2017).

Although the mocks are not drawn from N-body simulations, they have been calibrated to match one of the BigMultiDark (BigMD) (Klypin et al. 2016), a high resolution N-body simulation, with $3840^{3}$ particles coverig a volume of $\left(2500 h^{-1} M p c\right)^{3}$. The BigMD simulations were performed using GADGET-2 (Springel 2005), with $\Lambda$ CDM Planck cosmological constraints as a fiducial cosmology. $\Omega_{m}=0.307, \Omega_{b}=0.048206, \sigma_{8}=0.8288, n_{s}=0.96$, $H_{0}=100 h \mathrm{kms}^{-1} \mathrm{Mpc}^{-1}$ and $h=0.6777$. In Chuang et al. (2015) the authors showed that EZ-mocks are nearly indistinguishable from the full N-body solutions: they reproduce the power spectrum within $1 \%$, up to $k=0.65 h \mathrm{Mpc}^{-1}$. The mocks are created using a new efficient methodology based on the effective Zeldovich approximation approach including stochastic scale-dependent, non-local and nonlinear biasing contribution. The EZ mocks used for the current analysis are the light-cone catalogues, realized on 7 different snapshots at $z=0.9,1.1,1.3,1.5,1.6,1.7,2.0$. The full simulations incorporate the redshift evolution for $f, \sigma_{8}$, the BAO damping and the non-linear density and velocity effects.

In a companion paper (Ruggeri 2017) we will apply the weighting scheme to measure redshift-space distortions from the eBOSS DR 14 quasar data. In this paper, we validate the procedure and test for optimality. By fitting to the evolution with a model for bias and cosmology, we are able to fit simultaneously the evolution of the growth rate $f(z)$, the amplitude of the dark matter density fluctuations $\sigma_{8}(z)$ and the galaxy bias $b(z)$; breaking part of the degeneracy inherent in standard measurements of $f \sigma_{8}$ and $b \sigma_{8}$ when only one effective redshift is considered. We show that the redshift weighting scheme gives unbiased measurements.

The weights can be applied in both configuration or Fourier space. In this paper, we focus Fourier space, as there is some evidence that this provides stronger redshift space distortions (RSD) constrains, given the current scale limits within which the clustering can be modelled to a reasonable accuracy (Alam et al. 2016). In addition, the calculation of the power spectrum moments is significantly faster than the correlation function (Bianchi et al. 2015. Scoccimarro 2015). Working in Fourier-space requires a reformulation of the window selection to account for an evolving power spectrum.

The paper is organized as follows; Section 2 reviews the derivation of optimal weights, presenting two schemes that differ in the cosmological model to be tested. In Section 3 we review the redshift space power spectrum model at a single redshift; In Section 4 we model the power spectrum and the window function to obtain the redshift evolving power spectrum. In Section 5 we present the result of our analysis.

\section{OPTIMAL WEIGHTS}

We make use of two different sets of weights; the first explores deviations from the $\Lambda \mathrm{CDM}$ model by altering the evo- 
lution of $\Omega_{m}$ in redshift. This model ties together growth and geometry, but can also be used after fixing the expansion rate to match the prediction of the $\Lambda \mathrm{CDM}$ model. The second parametrizes the $f \sigma_{8}$ parameter combination measured by RSD, allowing for a more standard test of deviations from $\Lambda$ CDM. Here, the growth and geometry are artificially kept separate as $f \sigma_{8}$ only affects cosmological growth.

The weight functions act as a smooth window on the data and allow us to combine the information coming from the whole volume sampled. These weights are derived by minimising the error on the redshift space distortion measurements, as predicted by a simple Fisher matrix analysis (Ruggeri et al. 2017). Their derivation allows for the evolution with redshift of the cosmological parameters we want to estimate from the data. Optimizing the measurements of the parameters $\theta_{i}$ from the power spectrum moments $P_{j}$, we obtain the following weights,

$w(z)=C^{-1} \partial P_{j}(z) / \partial \theta_{i}$.

We assume the covariance matrix of $P, C$ to be parameter independent and, in absence of a survey window, to be described as

$C \sim\left(P_{\text {fid }}+1 / \bar{n}\right)^{2} 1 / d V$,

for each volume element, $d V$ within the survey. The weights can be seen as an extension of the FKP weights presented in Feldman et al. (1994), which have the form,

$w_{\mathrm{FKP}}(r)=\frac{1}{1+\bar{n}(r) P(k)}$,

by including the redshift component $\partial P_{j}(z) / \partial \theta_{i}$.

Note that the weights as they are reported in Eq. (1), aim to compress different measurements of the power spectrum across a range of different redshifts. In fact, we apply weights to each galaxy in order to avoid binning, by assuming $w_{\text {gal }}=\sqrt{w_{P}}$, which relies on the scale-dependence of the weights being smooth on the scale of interests for clustering.

\subsection{Optimal Weights for $\Omega_{m}$}

As described in Ruggeri et al. (2017), it is empirically convenient to test for deviations from the $\Lambda \mathrm{CDM}$ model by considering the evolution of the matter density with redshift. To do this, we consider a Taylor expansion up to second order about the fiducial model,

$\frac{\Omega_{m}(z)}{\Omega_{m, \text { fid }}(z)}=q_{0}\left[1+q_{1} y(z)+\frac{1}{2} q_{2} y(z)^{2}\right]$,

where $z_{p}$ is the pivot redshift and $y(z)+1 \equiv$ $\Omega_{m, \text { fid }}(z) / \Omega_{m, \text { fid }}\left(z_{p}\right)$. The $q_{i}$ parameters correspond to the first and second derivatives of $\left.\Omega_{m}(z)\right|_{z_{p}}$, evaluated at $z_{p}$, and incorporate potential deviations about the fiducial model $\Omega_{m, \text { fid }}$.

The choice of parameterising $\Omega_{m}$ (and hence the Hubble parameter, the angular diameter distance and the growth rate) in terms of $q_{0}, q_{1}$ and $q_{2}$ allows us to simultaneously investigate small deviations using a common framework; e.g. departures from a fiducial cosmology and geometry are accounted through the fiducial Hubble constant and angular diameter distance $H\left(\Omega_{m}\right), D_{A}\left(\Omega_{m}\right)$; further, modified

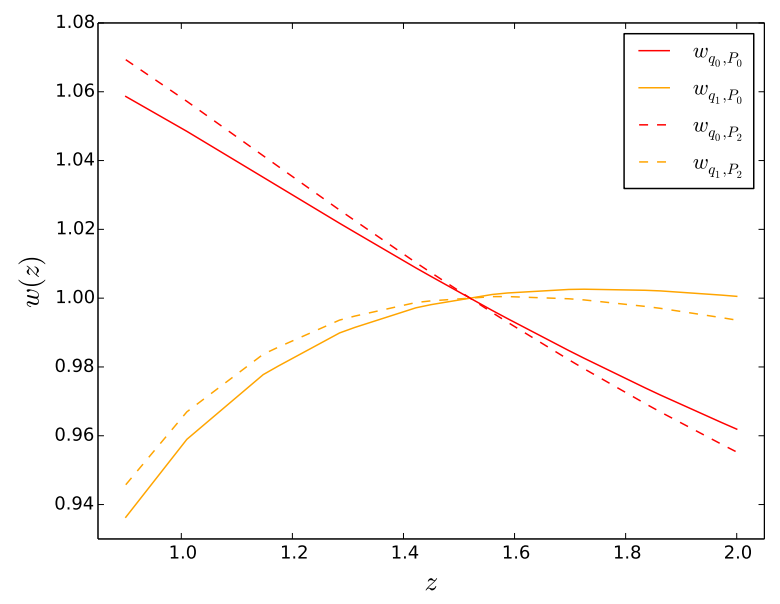

Figure 1. The weights for the monopole and quadrupole with respect to the $q_{i}$ parameters.

gravity models can be accounted through the growth rate, $f\left(\Omega_{m}\right)$.

By matching to the standard Friedman equation, we parametrize the redshift evolution of the Hubble parameter in term of $\Omega_{m}(z)$ as,

$H^{2}(z)=H_{0}^{2} \frac{\Omega_{m, 0}(1+z)^{3}}{\Omega_{m}(z)}$.

Assuming a flat Universe $\left(\Omega_{k}=0\right)$ in agreement with CMB measurements (Planck Collaboration et al. 2016), we have $\Omega_{\Lambda}(z)=1-\Omega_{m}(z)$. The subscript " 0 " denotes quantities evaluated at $z=0$. For simplicity of notation we omit the $q_{i}$ dependence from all the parameters: we refer to $\Omega_{m}\left(z, q_{i}\right)$ as $\Omega_{m}(z)$, and we denote with $\Omega_{m \text {,fid }}$ the fiducial $\Lambda$ CDM matter density.

For the scenarios considered, we assume the solution for the linear growth factor $D(z)$ and the dimensionless linear growth rate $f$ have the same dependence on $\Omega_{m}(z)$ as in the $\Lambda \mathrm{CDM}$ model,

$g(z) \equiv(1+z) D(z)=\frac{5 \Omega_{m}(z) H^{3}(z)}{2(1+z)^{2}} \int_{z}^{\infty} \mathrm{d} z^{\prime} \frac{\left(1+z^{\prime}\right)}{H^{3}\left(z^{\prime}\right)}$

$f(z)=-1-\frac{\Omega_{m}(z)}{2}+\Omega_{\Lambda}(z)+\frac{5 \Omega_{m}(z)}{2 g(z)}$.

Fig. 1 shows an example of the weights as derived in Ruggeri et al. (2017), that optimize the measurements of the $q_{i}$ parameters in a $\Lambda$ CDM fiducial background for a redshift-space power spectrum. Since each multipole contains information about $\Omega_{m}(z)$, our set of weights is derived to be optimal for the first two non-null moments of the power spectrum on the Legendre polynomial basis for each $q_{i}$ parameter. Continuous lines indicate the weights for the monopole with respect to $q_{0}$ (red line) and $q_{1}$ (orange line); dashed lines indicate the weights for the quadrupole with respect $q_{0}, q_{1}$ (red and orange lines). All the weights are normalized to be equal 1 at the pivot redshift;

\subsection{Optimal Weights for $f \sigma_{8}$}

RSD measurements constrain the amplitude of the velocity power spectrum, and its cosmological dependence in the lin- 
ear regime is commonly parameterized by the product of the two parameters $f$ and $\sigma_{8}$, which provides a good discriminator of modified gravity models (Song \& Percival 2009). We compare results obtained from the $\Omega_{m}$ parametrisation with those derived using a set of weights parametrised with respect to $\left[f \sigma_{8}\right](z)$. In an analogous way to the consideration in Section 2.1. we can expand $\left[f \sigma_{8}\right](z)$ about a fiducial model, so Eq. (4) becomes

$\left[f \sigma_{8}\right](z)=\left[f \sigma_{8}\right]_{f i d}(z) p_{0}\left(1+p_{1} x+p_{2} \frac{x^{2}}{2}\right)$

where $x \equiv\left[f \sigma_{8}\right]_{f i d}(z) /\left[f \sigma_{8}\right]_{f i d}\left(z_{p}\right)-1$. The $p_{i}$ parameters correspond to the first and second derivatives of $\left.\left[f \sigma_{8}\right](z)\right|_{z_{p}}$, evaluated at $z_{p}$, and incorporate potential deviations about the fiducial model $\left[f \sigma_{8}\right]_{\text {fid }}$.

We assume that in a $\Lambda \mathrm{CDM}$ model, $\left[f \sigma_{8}\right]_{\text {fid }}$ behaves as

$$
\begin{aligned}
{\left[f \sigma_{8}\right]_{\mathrm{fid}}(z) } & =\left[-1-\frac{\Omega_{m, \mathrm{fid}}(z)}{2}+\Omega_{\Lambda, \mathrm{fid}}(z)+\frac{5 \Omega_{m, \mathrm{fid}}(z)}{2 g_{\mathrm{fid}}(z)}\right] \\
& \times \sigma_{8,0} \frac{g_{\mathrm{fid}}(z)}{(1+z)^{2}},
\end{aligned}
$$

with $g_{\text {fid }}$, fiducial growth factor,

$g_{\text {fid }}(z)=\frac{5 \Omega_{m, \text { fid }}(z) H_{\text {fid }}^{3}(z)}{2(1+z)^{2}} \int_{z}^{\infty} \mathrm{d} z^{\prime} \frac{\left(1+z^{\prime}\right)}{H_{\text {fid }}^{3}\left(z^{\prime}\right)}$

The galaxy bias parameter is assumed to be independent of $f$ and $\sigma_{8}$. For simplicity, we consider $\left[b \sigma_{8}\right]$ to be independent from $\left[f \sigma_{8}\right]$ as well. Considering e.g. the galaxy monopole with respect to the linear matter power spectrum $P$,

$P_{0}=\left(\left[b \sigma_{8}\right]^{2}+\frac{2}{3}\left[b \sigma_{8}\right]\left[f \sigma_{8}\right](z)+\frac{1}{5}\left[f \sigma_{8}\right]^{2}(z)\right) P(k)$,

the dependence on the $p_{i}$ parameters is given only through $\left[f \sigma_{8}\right]$. We derive the set of weights by taking the derivative of $P_{0}, P_{2}, P_{4}$ with respect to $p_{1}, p_{2}, p_{3}$. For completeness we include the weights here as they were not included in Ruggeri et al. (2017).

$w_{i, q_{0}}=N_{i}, \quad w_{i, q_{1}}=N_{i} y, \quad w_{i, q_{2}}=N_{i} \frac{1}{2} y^{2}$,

where

$N_{0} \equiv\left(\frac{2}{3}\left[b \sigma_{8}\right]+\frac{2}{5}\left[f \sigma_{8}\right](z)\right)\left[f \sigma_{8}\right](z)$,

$N_{2} \equiv\left(\frac{4}{3}\left[b \sigma_{8}\right]+\frac{8}{7}\left[f \sigma_{8}\right](z)\right)\left[f \sigma_{8}\right](z)$

$N_{2} \equiv\left(\frac{16}{35}\left[f \sigma_{8}\right](z)\right)\left[f \sigma_{8}\right](z)$

A strong effect on the set of weights is caused by the assumptions we make for galaxy bias. If we set the bias as an unknown parameter, and we marginalize over it, then we cannot deduce any information about structure growth from the amplitude of the density power spectrum. This is the case for the expansion around $\left[f \sigma_{8}\right]$, where we considered $\left[b \sigma_{8}\right]$ and $\left[f \sigma_{8}\right]$ as independent parameters. However, if we constrain $b(z)$ to match a fiducial model, we will derive weights that make use of the information coming from the amplitude of the power spectrum. For the expansion around $\Omega_{m}$, we can choose whether or not to include this information.

\section{MODELLING THE ANISOTROPIC GALAXY POWER SPECTRUM AT A SINGLE REDSHIFT}

We model the power spectrum using perturbation theory (PT) up to 1-loop order. We include the non linear redshift space distortions effects as in Scoccimarro (2004) and Taruya et al. (2010) (TSN model),

$$
\begin{aligned}
P_{\mathrm{g}}(k, \mu)= & \exp \left\{-\left(f k \mu \sigma_{v}\right)^{2}\right\}\left[P_{\mathrm{g}, \delta \delta}(k)\right. \\
& +2 f \mu^{2} P_{\mathrm{g}, \delta \theta}(k)+f^{2} \mu^{4} P_{\theta \theta}(k) \\
& \left.+b^{3} A(k, \mu, \beta)+b^{4} B(k, \mu, \beta)\right],
\end{aligned}
$$

where $\mu$ is the cosine of the angle between the wave-vector $\mathbf{k}$ and the line of sight. $P_{\theta \theta}$ and $P_{\delta \theta}$ are the velocity-velocity and matter-velocity power spectra terms that correspond to the extended linear model of Kaiser (1987) as derived in Scoccimarro (2004). $\theta$ denotes the Fourier transform of the comoving velocity field divergence, $\theta(\mathbf{k}) \equiv-i \mathbf{k} \cdot \mathbf{u}(k)$ where $\nabla \mathbf{u}=-\nabla \mathbf{v} /[a f(a) H(a)]$ with velocity field $v$ and dimensionless linear growth rate $f$. The exponential term represents the damping due to the "Fingers of God" effect, where $\sigma_{v}$ denotes the velocity dispersion term, here treated as free parameter. The $A, B$ terms come from the TNS model which take into account further corrections due to the non linear coupling between the density and velocity fields (Taruya et al. 2010). Note that at linear level $P_{\theta \theta}=P_{\delta \theta}=P_{\delta \delta}$.

We model $P_{\mathrm{g}, \delta \delta}$ and $P_{\mathrm{g}, \delta \theta}$ as

$$
\begin{aligned}
P_{\mathrm{g}, \delta \delta}(k) & =b^{2} P_{\delta \delta}(k)+2 b_{2} b P_{b 2, \delta}(k)+2 b_{s 2} b P_{b s 2, \delta}(k) \\
& +2 b_{3 \mathrm{n} 1} b \sigma_{3}^{2}(k) P(k)+b_{2}^{2} P_{b 22}(k) \\
& +2 b_{2} b_{s 2} P_{b 2 s 2}(k)+b_{s 2}^{2} P_{b s 22}(k)+S, \\
P_{\mathrm{g}, \delta \theta}(k) & =b P_{\delta \theta}(k)+b_{2} P_{b 2, \theta}(k)+b_{s 2} P_{b s 2, \theta}(k) \\
& +b_{3 \mathrm{n} 1} \sigma_{3}^{2}(k) P(k),
\end{aligned}
$$

The bias is modelled following recent studies Chan et al. (2012); Baldauf et al. (2012) that showed the importance of non-local contributions. We account for those effects introducing as galaxy bias parameters: the linear $b$, second order local $b_{2}$, non local $b_{s 2}$, and the third order non-local $b_{3 \mathrm{nl}}$ bias parameters, and the constant stochasticity shotnoise term $S$. We numerically evaluate the non-linear matter power spectra, $P_{\delta \delta}, P_{\delta \theta}, P_{\theta \theta}$, at 1-loop order in standard perturbation theory (SPT) using the linear power spectrum input from CAMB (Lewis \& Bridle 2002).

In the current analysis we make use of the first three non-zero moments of the power spectrum, projected into an orthonormal basis of Legendre polynomials $\mathcal{L}_{\ell}(\mu)$ such that,

$P_{\ell}(k)=\frac{2 \ell+1}{2} \int_{-1}^{1} d \mu P(k, \mu) \mathcal{L}_{\ell}(\mu)$,

with the monopole $\ell=0$, quadrupole $\ell=2$ and hexadecapole $\ell=4$, respectively. In this paper we do not con- 
sider geometrical deviations and we are only concerned with growth measurements in a fixed background. However, we note that such deviations can be included as follows. The geometrical deviations from the fiducial cosmology can be included through the Alcock-Paczynski effect, (Alcock \& Paczynski 1979). Here, revised mode numbers $k^{\prime}, \mu^{\prime}$ for the cosmological model being tested, are related to those observed $k, \mu$ assuming the fiducial cosmology by the transformations

$$
\begin{aligned}
& k^{\prime}=\frac{k}{\alpha_{\perp}}\left[1+\mu^{2}\left(\frac{\alpha_{\perp}^{2}}{\alpha_{\|}^{2}}-1\right)\right]^{1 / 2} \\
& \mu^{\prime}=\frac{\mu \alpha_{\perp}}{\alpha_{\|}}\left[1+\mu^{2}\left(\frac{\alpha_{\perp}^{2}}{\alpha_{\|}^{2}}-1\right)\right]^{-1 / 2}
\end{aligned}
$$

where the scaling factors $\alpha_{\|}$and $\alpha_{\perp}$ are defined as

$$
\begin{gathered}
\alpha_{\|}=\frac{H^{\mathrm{fid}}(z)}{H(z)}, \\
\alpha_{\perp}=\frac{D_{A}(z)}{D_{A}^{\mathrm{fid}}(z)} .
\end{gathered}
$$

By applying the transformations of Eq. (21) to Eq. (19), the multipoles at the observed $k$ and $\mu$, relate to the power spectrum at the true variables $k^{\prime}$ and $\mu^{\prime}$ through

$P_{\ell}(k)=\frac{(2 \ell+1)}{2 \alpha_{\perp}^{2} \alpha_{\|}} \int_{-1}^{1} d \mu P_{g}\left(k^{\prime}, \mu^{\prime}\right) \mathcal{L}_{\ell}(\mu)$.

\section{MODELLING THE EVOLVING GALAXY POWER SPECTRUM}

\subsection{Redshift weighted multipoles without window function}

We model the redshift dependence of $f, \sigma_{8}, \alpha_{\|}$, and $\alpha_{\perp}$ as described above, and the bias evolution (see Sec. 4.3). In principle we can compute the weighted multipoles by integrating the power spectrum moments as given in Eq. 22 over redshift, including the redshift weighting,

$P_{\ell w_{\ell, q_{j}}}=\int d z P_{\ell}(k, z) w_{\ell, q_{j}}$.

However, by considering the power spectrum as a redshift evolving quantity we need to redefine the survey window function. It is easy to demonstrate that, by introducing an evolving power spectrum $P(k, z)$ into equation 2.1 .4 of Feldman et al. (1994), is no longer possible to derive the convolution relation 2.1.6 between the survey window function and the true power spectrum. We now consider how to allow for a generalized window function for redshift evolving power spectrum multipoles.

\subsection{Redshift weighted multipoles including the survey window effect}

We study the window function for the evolving power spectrum using a generalized Hankel transformation between power spectrum and correlation function moments, where the window applied is also decomposed into a set of multipoles. This is an extension of the work by Wilson et al. (2017) and Beutler et al. (2017), which presented a method to convolve model power spectra with the window function for a non-evolving power spectrum. We consider the case where the underlying correlation function $\xi$ is dependendent on both the separation $r=\left|r_{i}-r_{j}\right|$ (with $r_{i}$ and $r_{j}$ position of galaxies of each pair) and the mean redshift of each galaxy pair $\xi\left[r_{i}\left(z_{i}\right),\left|r_{i}-r_{j}\right|\right]$. Here we have assumed that cosmological evolution is negligible over the range of redshifts covered by every pair, so we can quantify the clustering of each using the correlation function at the mean redshift.

The multipole moments of the power spectrum in the local plane-parallel approximation can be written as,

$$
\begin{aligned}
\hat{P}_{\ell}(k)= & \frac{2 \ell+1}{2} \int d \mu_{k} \int \frac{d \phi}{2 \pi} \int d \mathbf{x}_{1} \int d \mathbf{x}_{2} e^{i \mathbf{k} \cdot \mathbf{x}_{1}} e^{-i \mathbf{k} \cdot \mathbf{x}_{2}} \\
& \left\langle\delta\left(\mathbf{x}_{1}\right) \delta\left(\mathbf{x}_{2}\right) W\left(\mathbf{x}_{1}\right) W\left(\mathbf{x}_{2}\right)\right\rangle \mathcal{L}_{\ell}\left(\hat{\mathbf{k}} \cdot \hat{\mathbf{x}}_{h}\right) \\
= & \frac{2 \ell+1}{2} \int d \mu_{k} \int \frac{d \phi}{2 \pi} \int d \mathbf{x}_{1} \int d \mathbf{s} \times \\
& \left(\sum_{L} \xi_{L}\left[s, z\left(x_{1}\right)\right] \mathcal{L}_{L}\left(\hat{\mathbf{x}}_{h} \cdot \hat{\mathbf{s}}\right)\right) \times \\
& W\left(\mathbf{x}_{1}\right) W\left(\mathbf{x}_{1}+\mathbf{s}\right) e^{-i \mathbf{k} \cdot \mathbf{s}} \mathcal{L}_{\ell}\left(\hat{\mathbf{k}} \cdot \hat{\mathbf{x}}_{h}\right),
\end{aligned}
$$

where $\int d \mu_{k}$ is the integral over all the possible cosine angles between $\hat{\mathbf{k}}$ and $\hat{\mathbf{x}}_{h}$ and $W$ defines the mask. $\xi_{L}$ denotes the correlation function moments in the Legendre basis. Note that Eq. 24 differs from equation A.16 in Beutler et al. (2017), only in the $\xi_{L}\left[s, z\left(x_{1}\right)\right]$ term; for a single redshift slice we would only have $\xi_{L}(s)$. We make use of the relations,

$e^{-i \mathbf{k} \cdot \mathbf{s}}=\sum_{p}(i)^{p}(2 p+1) j_{p}(k s) \mathcal{L}_{p}(\hat{\mathbf{k}} \cdot \hat{\mathbf{s}})$,

$\int d \mu_{k} \int \frac{d \phi}{2 \pi} \mathcal{L}_{\ell}\left(\hat{\mathbf{k}} \cdot \hat{\mathbf{x}}_{h}\right) \mathcal{L}_{p}(\hat{\mathbf{k}} \cdot \hat{\mathbf{s}})=\frac{2}{2 \ell+1} \mathcal{L}_{\ell}\left(\hat{\mathbf{s}} \cdot \hat{\mathbf{x}}_{h}\right) \delta_{\ell p}$

which, when combined with Eq. 24, give

$$
\begin{gathered}
\hat{P}_{\ell}(k)=i^{\ell}(2 \ell+1) \int d \mathbf{x}_{1} \int d \mathbf{s} \sum_{L} \xi_{L}\left[s, z\left(x_{1}\right)\right] j_{\ell}(k s) \times \\
W\left(\mathbf{x}_{1}\right) W\left(\mathbf{x}_{1}+\mathbf{s}\right) \mathcal{L}_{\ell}\left(\hat{\mathbf{x}}_{h} \cdot \hat{\mathbf{s}}\right) \mathcal{L}_{L}\left(\hat{\mathbf{x}}_{h} \cdot \hat{\mathbf{s}}\right) .
\end{gathered}
$$

Substituting the Bailey relation, $\mathcal{L}_{\ell} \mathcal{L}_{p}=\sum_{t} a_{p t}^{\ell} \mathcal{L}_{t}$, Eq. 27. becomes,

$$
\begin{aligned}
\hat{P}_{\ell}(k)= & i^{\ell}(2 \ell+1) \int d \mathbf{x}_{1} \int d \mathbf{s} \sum_{L} \xi_{L}\left[s, z\left(x_{1}\right)\right] j_{\ell}(k s) \times \\
& W\left(\mathbf{x}_{1}\right) W\left(\mathbf{x}_{1}+\mathbf{s}\right) \mathcal{L}_{\ell}\left(\hat{\mathbf{x}}_{h} \cdot \hat{\mathbf{s}}\right) \sum_{t} a_{L t}^{\ell} \mathcal{L}_{t}\left(\hat{\mathbf{x}}_{h} \cdot \hat{\mathbf{s}}\right) . \\
= & i^{\ell}(2 \ell+1) \int 2 \pi s^{2} d s j_{\ell}(k s) \sum_{L} \sum_{t} a_{L t}^{\ell} \int d \mu_{s} \int \frac{d \phi}{2 \pi} \\
& \times \int d \mathbf{x}_{1} \xi_{L}\left[s, z\left(x_{1}\right)\right] W\left(\mathbf{x}_{1}\right) W\left(\mathbf{x}_{1}+\mathbf{s}\right) \mathcal{L}_{t}\left(\hat{\mathbf{x}}_{h} \cdot \hat{\mathbf{s}}\right)
\end{aligned}
$$

At this stage, in contrast to Eq. A.19 in Beutler et al. (2017), we cannot bring $\xi_{L}$ out of the integral over $x_{1}$. Since we are not able to decouple the mask from $\xi$, in principle, we would have to compute the integral over $x_{1}$ for every model $\xi$ fitted to the data. However we can reduce drastically the computational time required by assuming that $\xi$ is well behaved such that we can split the integral over $x_{1}$ into a sum over a small number of $x_{i}$ ranges. This is different 
from measuring the clustering in shells - we are still calculating and modelling the power spectrum as a continuously weighted function calculated using every galaxy pair; we are simply making an assumption about the smooth behaviour in redshift of the expected clustering.

$$
\begin{aligned}
\hat{P}_{\ell}(k)= & i^{\ell}(2 \ell+1) \int 2 \pi s^{2} d s j_{\ell}(k s) \sum_{L} \sum_{t} a_{L t}^{\ell} \int d \mu_{s} \int \frac{d \phi}{2 \pi} \\
& \times \sum_{i} \int_{x_{i}} d \mathbf{x}_{1} \xi_{L}\left(s, z\left(x_{i}\right)\right) W\left(\mathbf{x}_{i}\right) W\left(\mathbf{x}_{i}+\mathbf{s}\right) \mathcal{L}_{t}\left(\hat{\mathbf{x}}_{h} \cdot \hat{\mathbf{s}}\right) .
\end{aligned}
$$

Assuming that $\xi_{L}\left(s, z\left(x_{i}\right)\right)$ is constant over each sub-integral range $x_{i}$ we can take it out of the integrals,

$$
\begin{aligned}
& \hat{P}_{\ell}(k)=i^{\ell}(2 \ell+1) \int 2 \pi s^{2} d s j_{\ell}(k s) \sum_{L} \sum_{t} a_{L t}^{\ell} \times \\
& \sum_{i} \xi_{L}\left(s, z\left(x_{i}\right)\right) \int d \mu_{s} \int \frac{d \phi}{2 \pi} \int_{x_{i}} d \mathbf{x}_{i} W\left(\mathbf{x}_{i}\right) W\left(\mathbf{x}_{i}+\mathbf{s}\right) \mathcal{L}_{t}\left(\hat{\mathbf{x}}_{h} \cdot \hat{\mathbf{s}}\right),
\end{aligned}
$$

and redefine the sub-window function multipoles $W_{p, z_{i}}^{2}(s)$ for $p=0,2,4$.. as

$$
\begin{aligned}
W_{p, z_{i}}^{2}(s)= & \frac{2 p+1}{2} \int d \mu_{s} \int \frac{d \phi}{2 \pi} \int_{z_{i}} d \mathbf{x}_{i} \\
& \times W\left(\mathbf{x}_{i}\right) W\left(\mathbf{x}_{i}+\mathbf{s}\right) \mathcal{L}_{p}\left(\mu_{s}\right) .
\end{aligned}
$$

Using the definition of the sub-window function multipoles of Eq. 31, we can write Eq. 30 to be

$$
\begin{aligned}
\hat{P}_{\ell}(k)= & i^{\ell}(2 \ell+1) \int 2 \pi s^{2} d s j_{\ell}(k s) \times \\
& \sum_{L} \sum_{t} \frac{2}{2 t+1} a_{L t}^{\ell} \sum_{i} \xi_{L}\left(s, z_{i}\right) W_{t, z_{i}}^{2}(s) .
\end{aligned}
$$

which generalizes Eq. A.23 in Beutler et al. (2017) to the case of a redshift-evolving power spectrum.

In this study we make use of the redshift weights to compute the weighted multipoles $\hat{P}_{\ell, w_{j}}$ : we include the weights in the model by inserting them in the sub-window multipoles. As these are calculated using a pair-counting approach we apply the weights to the pairs as for the data. The binning of the mask does not affect our ability to use continuous redshift weighting on the data and in the model. Thus, we do not bin our redshift weights. However, we do assume that the weights are scale independent, so they can be applied to the galaxies assuming $w_{g}=\sqrt{w_{P}}$.

Note that we include a fiducial model for the evolution of the power spectrum when calculating the weights; however, even in the case that the fiducial model does not match accurately the data, the weights would not bias the measurements; instead we would have that the weights we are using are not optimal.

\subsection{Bias evolution}

Differently from the other quantities the galaxy bias, $b$, requires a more careful study before including it in the evolving power spectrum: $b(z)$ strongly depends on the targets and there is not a cosmological constraint; for this reason we should allow for more freedom in the form that it can take.

In Ruggeri (2017) we compared the weights for different $b(z)$ relations and showed that the weights are not significantly sensitive to the different $b(z)$ considered;

The fitting formula for the linear bias parameter of the quasar sample suggests that the linear bias redshift evolves as, (Laurent et al. 2017),

$b(z)=0.53+0.29(1+z)^{2}$.

We model the evolution of $b$ about the pivot redshift times $\sigma_{8}$ as,

$b \sigma_{8}(z)=b \sigma_{8}\left(z_{\mathrm{p}}\right)+\partial b \sigma_{8} /\left.\partial z\right|_{z_{p}}\left(z-z_{\mathrm{p}}\right)+\ldots$

We neglect the redshift dependence for the non linear bias parameter $b_{2}$, so we assume this is constant with redshift, $b_{2} \sigma_{8}\left(z_{\text {piv }}\right)$. We fix the 2nd-order non-local bias, $b s_{2}$ and 3rdorder non-local bias, $b_{3 n l}$ terms to their predicted values according to non-local Lagrangian models, Chan et al. 2012, Baldauf et al. 2012),

$$
\begin{aligned}
b_{s 2} & =-\frac{4}{7}(b-1), \\
b_{3 n l} & =\frac{32}{315}(b-1) .
\end{aligned}
$$

\section{FITTING TO THE MOCK DATA}

\subsection{Power spectrum measurement}

To compute the power spectrum moments with respect to the line of sight (LOS), we make use of the estimator introduced in Bianchi et al. (2015). This fourier transform (FT) - based algorithm uses multiple FTs to track the multipole moments, in the local plane-parallel approximation where we have a single LOS for each pair of galaxies. This estimator has been already used in recent analysis (Beutler et al. 2017), that confirmed the advantages of using such decomposition: it reduces the computational time from $N^{2}$ associated to naive pair counting analysis Yamamoto et al. (2006) to $\sim N \log N$.

Redshift weights are included in the estimator, by defining the weighted galaxy number density as $n_{g}(\mathbf{r}) w$. As discussed in Section 2 we have derived the galaxy weights from the square-root of the power spectrum weights, under the assumption that the scale dependence in the weights is smooth compared to the scale of interest for our clustering measurements.

The result is a set of weighted multipoles, $P_{0,2, w_{0,1,2}}$, where each $P_{i, w_{j}}$ corresponds to a particular set of weights that optimizes each of the $q_{i}$ or $p_{i}$ measurement, i.e. for the set of weights $w_{i, q_{j}}$ (or $w_{i, p_{j}}$ for the $f \sigma_{8}$ weights) functions and we build a data vector $\Pi$ as,

$\Pi^{T}=\left(P_{0, w_{0}, q_{0}}, P_{0, w_{0}, q_{1}}, P_{0, w_{0}, q_{2}} \ldots P_{2, w_{2}, q_{2}}\right)^{\mathrm{T}}$.

\subsection{Covariance matrix estimation}

We evaluate the covariance matrix for the data vector $\Pi^{T}$ using 1000 EZ mock described in Section 1. For each mock, we compute the weighted monopole and quadrupole moments for each set of optimal redshift weights, for $n_{b}=10 k$-bins 
in the range of $k=0.01-0.2 h \mathrm{Mpc}^{-1}$. From these, we derive the covariance matrix as

$$
\begin{aligned}
C=\frac{1}{N_{\mathrm{T}}-1} \sum_{n=1}^{N_{\mathrm{T}}}\left[P_{n, \ell, w_{\ell, q_{t}}}\left(k_{i}\right)-\hat{P}_{\ell, w_{\ell, q_{t}}}\left(k_{i}\right)\right] \\
\times\left[P_{n, \ell, w_{\ell, q_{t}}}\left(k_{j}\right)-\hat{P}_{\ell, w_{\ell, q_{t}}}\left(k_{j}\right)\right],
\end{aligned}
$$

where $N_{T}=1000$ is the number of mock catalogues, $w_{\ell, q_{t}}$ denotes each set of weights for each parameter $q_{t}$ (or $p_{t}$ ) and $\hat{P}_{\ell, w_{\ell, q_{t}}}\left(k_{i}\right)=\frac{1}{N_{\mathrm{T}}} \sum_{n=1}^{N_{\mathrm{T}}} P_{n, \ell, w_{\ell, q_{t}}}\left(k_{i}\right)$.

Note that when inverting the covariance matrix we include the Hartlap factor (Hartlap et al. 2007) to account for the fact that $C$ is inferred from mock catalogues.

\subsection{Maximising the Likelihood}

Since each weighted multipole $P_{i, w_{i, q_{j}}}$ is optimized with respect to a particular piece of information (e.g. $\Omega_{m}[z]$ ), we jointly fit all three $q_{i}$ (or $p_{i}$ ) parameters simultaneously. We compare the measured $\Pi^{T}$ to modelled weighted power spectra multipoles, convolved with the window function as explained in Section 4.2 . We assume a Gaussian likelihood and minimize

$\chi^{2} \propto\left(\Pi-\Pi_{\text {model }}\right)^{\mathrm{T}} C^{-1}\left(\Pi-\Pi_{\text {model }}\right) ;$

Where $\Pi_{\text {model }}$ refers to the window convolved $P_{i, w_{i, q_{j}}}$. The $C^{-1}$ term corresponds to the joint covariance derived in Eq. (37). We repeat the fit for both the $\Omega_{m}$ and $f \sigma_{8}$ optimized sets of weights.

In the current analysis we limit ourselves to linear order deviations about our fiducial $\Lambda$ CDM model, for both $f \sigma_{8}(z)$ and $\Omega_{m}(z)$ described in Sections 2.1 and 2.2 since the data cannot capture second-order deviations. We discuss this further in Section 7

\section{MEASURING RSD WITH THE EVOLVING GALAXY POWER SPECTURM}

The fits presented in this section are performed using a Monte Carlo Markov Chain (MCMC) code, implemented to efficiently account for the degeneracies between the parameters; in all the fit performed we select a range between $k=0.01-0.2 h \mathrm{Mpc}^{-1}$. For each scenario explored we run 10 independent chains, satisfying the Gelman-Rubin convergence criteria (Gelman \& Rubin 1992) with the requirement of $R-1<10^{4}$; where $R$ corresponds to the ratio between the variance of chain mean and the mean of chain variances. All the results presented are obtained after marginalizing on the full set of parameters, including the nuisance parameters (shotnoise and velocity dispersion). All the contour plots are produced using the public getdist libraries ${ }^{3}$

We fit the weighted monopole and quadrupole computed on a subset of $20 \mathrm{EZ}$ mocks, for both the $\Omega_{m}$ and the $f \sigma_{8}$-optimized weights, while keeping the distance-redshift relation fixed to the fiducial cosmology, i.e. $\alpha_{\|}=\alpha_{\perp}=1$.

We do not consider the full set of $1000 \mathrm{EZ}$ mocks for the following reasons; first we are limited by the EZ-mock

${ }^{3}$ http://getdist.readthedocs.io/ accuracy in describing non linearities in galaxy bias and velocities; further by the accuracy in the light-cone describing the redshift evolution for $f \sigma_{8}$ which is included as a step function. Thus we do not believe that the mocks supports us looking at deviations from the model at better accuracy than this. However, the error on our constraints is still $1 / \sqrt{20}$ smaller than what we expect on the eBOSS quasars constraints. The analyisis has been performed on different subset of 20 mocks out of the 1000 available to verify that the outcomes do not depend on a particular subsample choice.

Our analysis is presented as follow; in 6.1 we present the result obtained with the $\Omega_{m}$ weights fitting for $q_{0}, q_{1}$, $b \sigma_{8}\left(z_{\mathrm{p}}\right), \partial b \sigma_{8} /\left.\partial z\right|_{z_{p}}, b_{2}, \sigma_{v}$, and shotnoise $S$. In parallel we present the fit for $p_{0}, p_{1}, b \sigma_{8}\left(z_{\mathrm{p}}\right), \partial b \sigma_{8} /\left.\partial z\right|_{z_{p}}, b_{2}, \sigma_{v}$ when applying the $f \sigma_{8}$ weights.

In 6.2 we investigate the impact of the bias assumption on the contraints, showing a comparison between bias evolving and constant with redshift.

In 6.3 we compare the results obtained with the redshift weights approach with the analysis performed considering one constant redshift slice i.e. considering all the parameters $\left(f \sigma_{8}, b \sigma_{8}, \sigma_{v}, b_{2}, S\right)$ in the power spectra at their value at the pivot redshift $z=1.55$ and applying FKP weights only (for simplicity of the notation from now on we refer to this as traditional analysis).

Differently from Zhu et al. (2016), we compare the redshift weights analysis with the standard analysis used for previous RSD measurements (see e.g. Beutler et al. (2017)) rather than testing the weights $w_{q, i}, w_{p, i}=1$. The main focus of this work is to test that our analysis is not biased by introducing evolution in the power spectrum and in the window function. We rely on the Fisher matrix theory correctly selecting the set of weights optimal with respect to the $q_{i}, p_{i}$ errors.

\subsection{Redshift weights fit}

Fig. 2 shows the posterior likelihood distributions from the analysis performed with the set of redshift weights optimized to constrain $\Omega_{m}(z)$ (blue contour plots), using the monopole and the quadrupole; we fit for $q_{0}, q_{1}$ which describe up to linear order deviations in the evolution of $\Omega_{m}(z)$ according to $\Lambda$ CDM model; we also vary the galaxy bias parameters modelled as in section 4.3 , while we fix the 2nd-order nonlocal bias, $b s_{2}$ and 3rd-order non-local bias, $b_{3 n l}$ terms as shown in Eq. 35 . To summarize we fit for 7 parameters: $q_{0}$, $q_{1}, b \sigma_{8}\left(z_{\mathrm{p}}\right), \partial b \sigma_{8} /\left.\partial z\right|_{z_{p}}, b_{2} \sigma_{8}\left(z_{p}\right), \sigma_{v}$, and shotnoise $S$.

Fig 3 presents the results of the analysis while using the set of redshift weights optimized to constrain $f \sigma_{8}(z)$, as introduced in Sec. 2.2 the structure is the same as in Fig. 2 We fit for $p_{0}, p_{1}$ to constrain $f \sigma_{8}(z)$ deviations about the fiducial $f \sigma_{8}(z)$ according to $\Lambda \mathrm{CDM}$; we also fit for $b \sigma_{8}\left(z_{\mathrm{p}}\right)$, $\partial b \sigma_{8}(z) / \partial z, b_{2} \sigma_{8}\left(z_{p}\right), \sigma_{v}, S, 7$ parameters in total as for the other set of weights.

The resulting posteriors in both Figures 2 and 3 show a correlation between the zero order parameters, $q_{0}\left(p_{0}\right)$ and $b \sigma_{8}\left(z_{\mathrm{piv}}\right)$, of magnitude of $\sim 0.5$. We also detect a relevant anti-correlation $\sim-0.4$ between the slope parameter $q_{1}\left(p_{1}\right)$ and the gradient $\partial b \sigma_{8}(z) / \partial z$. We tested and confirmed that those correlations lead to a mild dependency between the assumed bias model (linear and non linear in $k$ and in $z$ ) and the slope parameter $q_{1}\left(p_{1}\right)$ without however affecting 


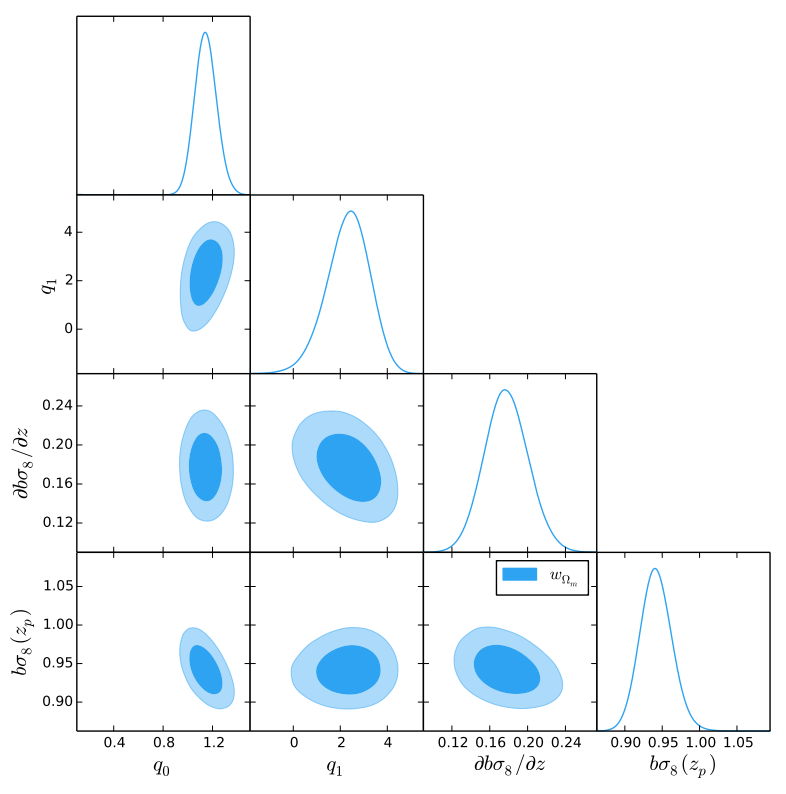

Figure 2. Likelihood distributions for the analysis of the average of $20 \mathrm{EZ}$ mock. We show the results for $q_{0}, q_{1}, b \sigma_{8}\left(z_{p}\right), \partial b \sigma_{8} / \partial z$, marginalized over the full set parameters (including $b_{2} \sigma_{8}\left(z_{p}\right), \sigma_{v}$, $S$ not dispayed here). We multi-fit two weigthed monopoles and two weigthed quadrupoles (one for each weight function $\left(w_{0, p_{i}}\right.$, $w_{2, p_{i}}$ ) The fitting range is $k=0.01-0.2 h \mathrm{Mpc}^{-1}$ for both the monopole and quadrupole.

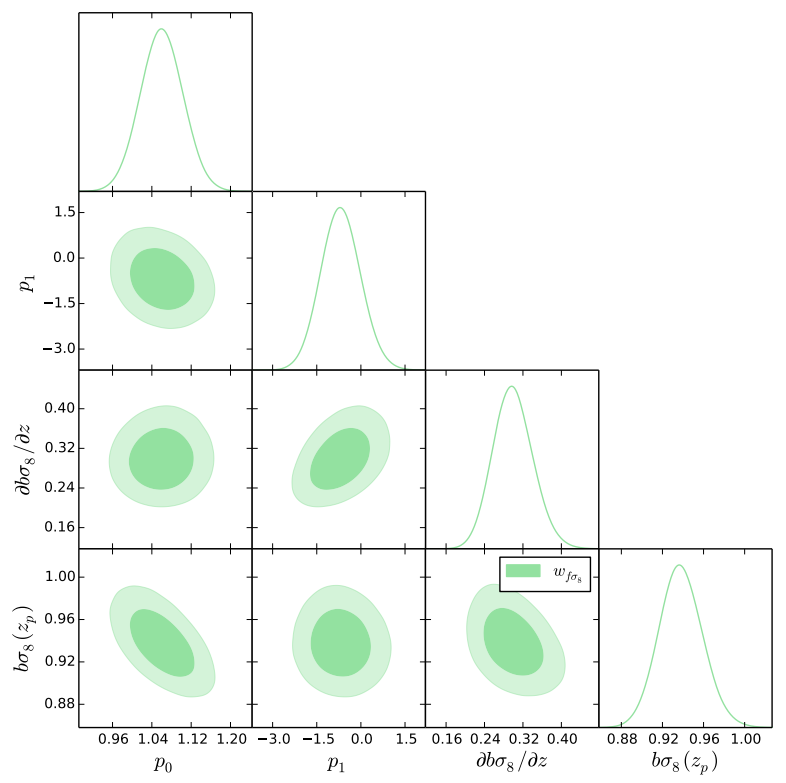

Figure 3. Likelihood distributions for the analysis of the average of $20 \mathrm{EZ}$ mock. We show the results for $p_{0}, p_{1}, b \sigma_{8}\left(z_{p}\right), \partial b \sigma_{8} / \partial z$, marginalized on the full set parameters (including $b_{2} \sigma_{8}\left(z_{p}\right), \sigma_{v}$, $S$ not dispayed here). We multi-fit two weigthed monopoles and two weighted quadrupoles (one for each weight function $\left(w_{0, p_{i}}\right.$, $w_{2, p_{i}}$ ) The fitting range is $k=0.01-0.2 h \mathrm{Mpc}^{-1}$ for both the monopole and quadrupole.

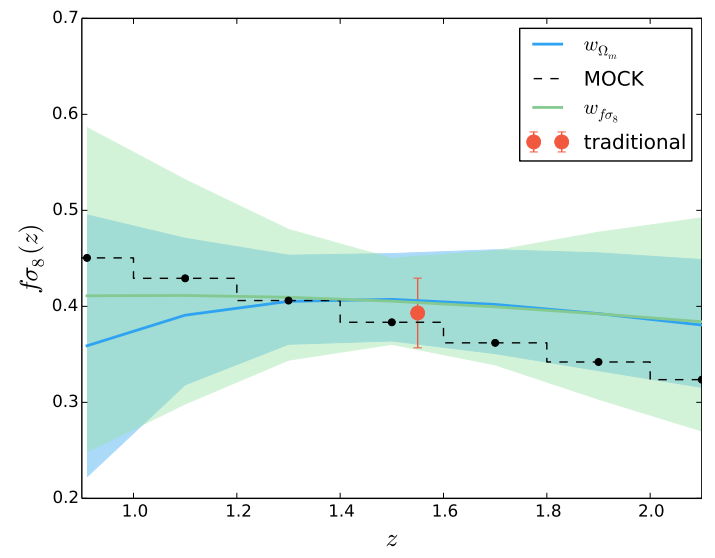

Figure 4. The reconstructed evolution of $f \sigma_{8}$ and $68 \%$ confidence level regions using the average of 20 mocks; blue shaded region shows the constraint on the evolution of $f \sigma_{8}$ obtained by the fit of $\Omega_{m}\left(z, q_{i}\right)$ using the $w_{\Omega_{m}}$ optimal weights and deriving at each redshift $f\left[\Omega_{m}\left(z, q_{i}\right)\right]$ times $\sigma_{8}\left[\Omega_{m}\left(z, q_{i}\right)\right]$; green shaded region shows the resulting evolution when fitting for $f \sigma_{8}\left(z, p_{i}\right)$ at each redshift. The red point indicates the results obtained when performing the traditional analysis, with $z_{\text {piv }}=1.55$.

(within $\sim 1 \sigma$ ) the constraints on $f \sigma_{8}$. In 6.2 we illustrate this in more details. Due to the stepwise implementation of the growth rate and bias model in the mocks, the fiducial values of $q_{0}, q_{1}\left(p_{0}, p_{1}\right)$ are not well defined. Therefore, we do not display an expected value for $p_{i}$ and $q_{i}$ as those cannot be inferred from the $f \sigma_{8}$ evolution included as a non-smooth step function in the mocks. However, within 1 to 2 sigma we recover the smooth $\Lambda \mathrm{CDM}$ expectation values of $q_{0}=1$ and $q_{1}=0$.

Fig. 4 shows the redshift evolution reconstructed from $p_{0}, p_{1}$, (green shaded regions), compared with the evolution reconstructed from the $q_{0}, q_{1}$ (blue shaded regions). The red point indicates the constraints at one single redshift (traditional analysis, with $z=1.55)$ for $f \sigma_{8}$. We overplot the evolution of $f \sigma_{8}(z)$ as accounted in the mock lightcone (black dashed line). The plot shows that the $f \sigma_{8}$ evolution obtained for both the $\Omega_{m}$ and $f \sigma_{8}$ weighting schemes is fully consistent with the cosmology contained in the mock and in full agreement with the constraints coming from the traditional analyis. For both parametrizations the errors obtained at the pivot redshift is comparable with the error we get from the traditional analysis. Note that the error from redshift weigthing analysis comes from the marginalization over a set of 7 parameters in constrast to the traditional analyisis limited to only 5 free parameters.

Away from from the pivot redshift, the errors becomes larger for both parametrizations. At these redshifts, the major contribution to the error comes from the slope constraints $\left(q_{1}, p_{1}\right)$ and the $\mathrm{S} / \mathrm{N}$ is lowered due to the low number density $n(z)$, (Ata et al. 2017). For both parametrizations, the slope parameters are degenarate with the non linear bias parameters.

In 6.1 we modelled the bias evolution with a Taylor expansion up to linear order about the pivot redshift (see Eq. 4.3. Fig. 5 shows the $b \sigma_{8}(z)$ evolution measured using the $\Omega_{m}$ and $f \sigma_{8}$ weighting schemes (blue and green shaded regions). We reconstruct $b \sigma_{8}(z)$ at the different redshifts from the fit of $b \sigma_{8}\left(z_{p}\right)$ and $\partial b \sigma_{8}(z) / \partial z$. We overplot the evolution 


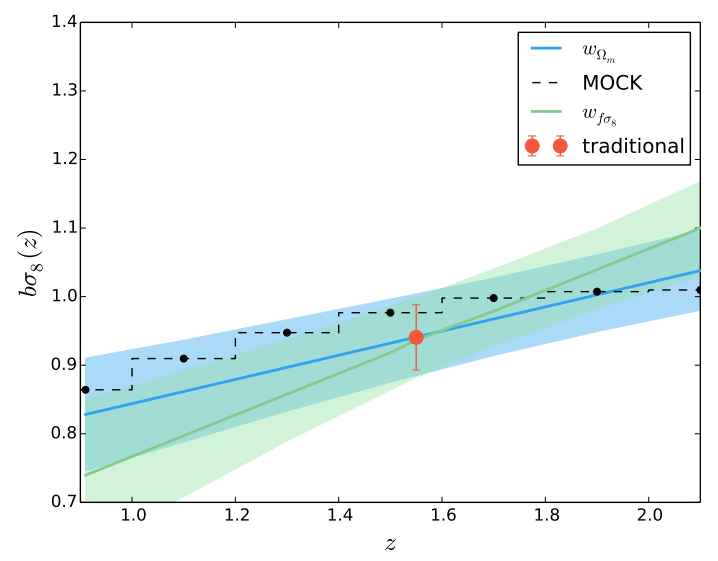

Figure 5. The reconstructed evolution for $b \sigma_{8}(z)$ and $68 \%$ confidence level regions using the average of 20 mocks; we fit the evolution for $b \sigma_{8}$, modelled as a Taylor expansion about the pivot redshift, up to linear order. Blue shaded regions show the evolution of $b \sigma_{8}$ through the fit of $b \sigma_{8}\left(z_{p}\right), \partial b \sigma_{8}(z) / \partial z$, obtained for the $\Omega_{m}\left(q_{i}\right)$ analysis; green shaded regions show the analogous resulting $b \sigma_{8}(z)$ when fitting for $f \sigma_{8}\left(z, p_{i}\right)$ at each redshift. The red point indicates the results obtained for $f \sigma_{8}\left(z_{\text {piv }}\right)$ when performing the traditional analysis.

of $b \sigma_{8}(z)$ as included in the mocks (black dashed line). The red point indicates the constraints obtained by using the traditional analysis; we find full agreement at the pivot redshift between the three different analysis and within $1 \sigma$ of the value included in the mocks. The bias depends significantly on redshift and in the mocks is modelled as a step function, which leads to small discrepancies with respect to both the constant and linear evolution in $b \sigma_{8}$. We redid the fit extending the analysis to second order in bias and found consistent results but with error too large to see any improvements (high degeneracy). For the purpose of fitting eBOSS quasar sample this is more than enough and we leave for future work a more careful study of the bias effects /evolution to be performed on more accurate nbody mocks. This is discussed further in section 7

\subsection{Constant bias vs evolving bias}

We now investigate how a particular choice for the bias evolution in redshift can affect and impact the constraints on $f \sigma_{8}(z)$. To do this, we repeat the analysis as presented in 6.1 using the $\Omega_{m}$ and $f \sigma_{8}$ weights, we model $\Omega_{m}(z)$ and $f \sigma_{8}(z)$ in the same way as in 6.1. but now assuming that the bias is constant with redshift i.e we set $\partial b \sigma_{8}(z) / \partial z=0$.

In Figures 6 7 we show the comparison between the results obtained with the constant bias. We display the posterior likelihood for all the quantities evaluated at the pivot redshift, $f \sigma_{8}\left(z_{p}\right) b \sigma_{8}\left(z_{p}\right), \sigma_{v}, b_{2}, S$. In Figure 6, blue contours show the likelihood distributions obtained when using the $\Omega_{m}$ weights and considering $b \sigma_{8}$ evolving as in Eq. 4.3. Dark blue contours indicate the constraints obtained when considering $\partial b \sigma_{8}(z) / \partial z=0$. In Figure 7 we present the analogous results when using the $f \sigma_{8}$ parametrization; green contours show the likelihood distributions obtained when using the $f \sigma_{8}$ weights considering the bias evolving as in Eq. 4.3. Dark green contours correspond to the constraints obtained when we set $\partial b \sigma_{8}(z) / \partial z$ equal to zero. The

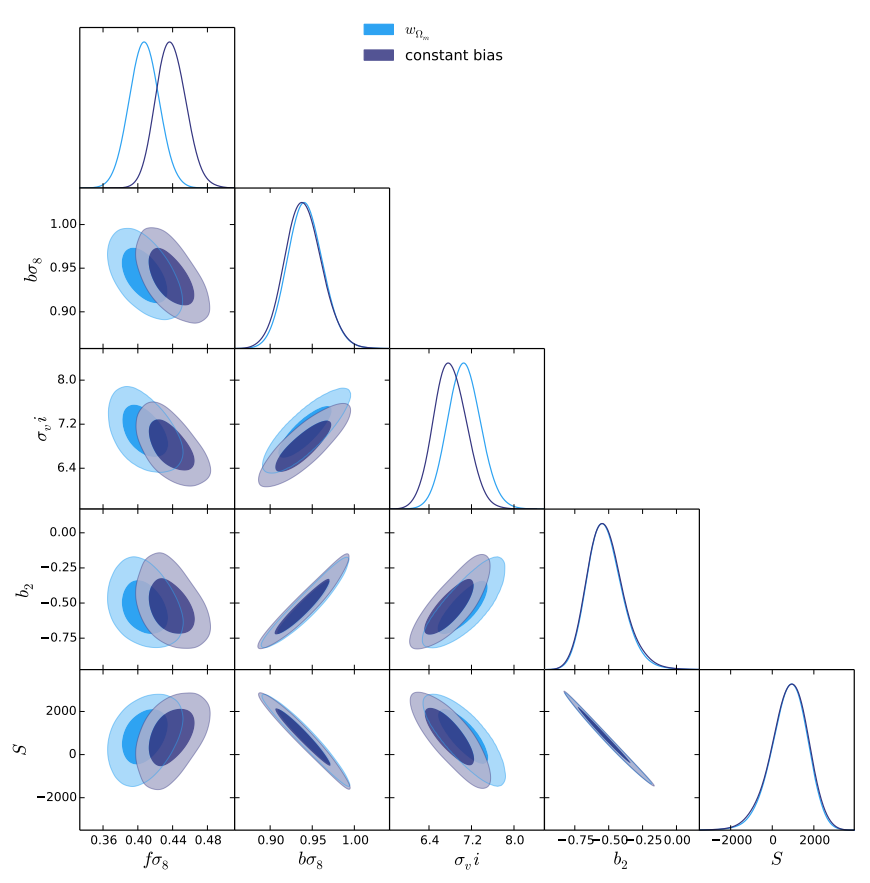

Figure 6. Comparison between evolving and constant bias for the $\Omega_{m}$ - weights analysis. Blue likelihood contours indicate the constraints obtained when fitting for $b \sigma_{8}\left(z_{p}\right)$ and $\partial b \sigma_{8}(z) / \partial z$; dark blue contours indicate the constraints obtained when setting $\partial b \sigma_{8}(z) / \partial z=0$ and fitting only for $b \sigma_{8}\left(z_{p}\right)$.

results obtained from the different models are consistent, but, whereas the constraints for $b \sigma_{8}\left(z_{p}\right)$ remain unchainged there is an evident impact on the $f \sigma_{8}$ constraints at the pivot redshift. Forcing the bias to be constant with redshift lead to an higher value for $f \sigma_{8}$. This should be kept in mind for future work when higher precision is expected: a careful study/treatment of the bias is required in order to be sure not to bias the constraints on the growth.

\subsection{Weights vs no Weights}

We compare the analysis performed using the redshift weights approach, as presented in 6.1 with the traditional analysis at one constant redshift.

The traditional analysis makes use of the power spectrum moments, modelled as in Sec. 3. to constrain $f \sigma_{8}$ and $b \sigma_{8}$ at one single epoch which corresponds to the effective redshift of the survey $(z=1.55)$. We do the comparison for both the $\Omega_{m} f \sigma_{8}$ weigthing schemes;

Figure 8 shows the comparison between the redshift weights analysis for $\Omega_{m}$ (blue contours), $f \sigma_{8}$ (green contours) and the constant redshift analysis (brown contours). In order to make the comparison between the three different analysis we infer from the MCMC chains of $q_{i}$ and $p_{i}$, the $f \sigma_{8}\left[z, \Omega_{m}\left(q_{i}\right)\right]$ and $f \sigma_{8}\left(z, p_{i}\right)$ valued at the pivot redshifts. We then compare those values with the $f \sigma_{8}\left(z_{p}\right), b \sigma_{8}\left(z_{p}\right)$ as obtained from the traditional analysis. The last two panels in Fig. 8 show that we recover the same value for $b_{2}$, and $S$ where the evolution in redshift is not considered in all the three different analysis; the other constraints on $f \sigma_{8}, b \sigma_{8}$ and $\sigma_{v}$ are fully consistent within $\sim 1 \sigma$. 


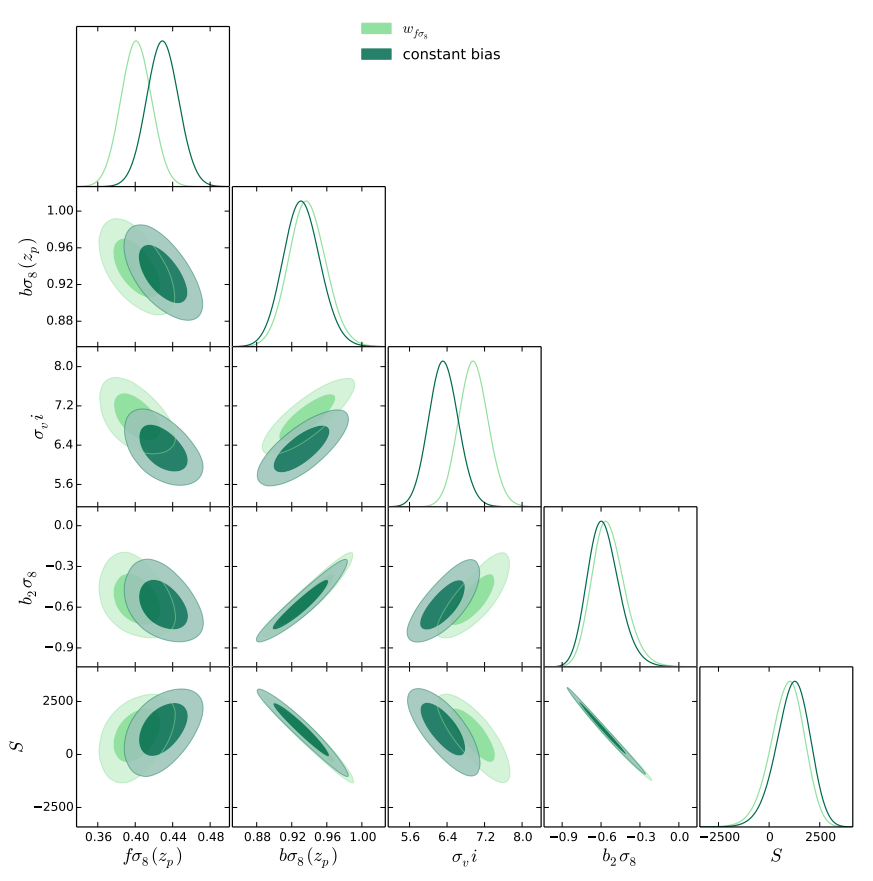

Figure 7. Comparison between evolving and constant bias for the $f \sigma_{8}$-weights analysis. Green likelihood contours indicate the constraints obtained when fitting for $b \sigma_{8}\left(z_{p}\right)$ and $\partial b \sigma_{8}(z) / \partial z$; dark green contours indicate the constraints obtained when setting $\partial b_{8}(z) / \partial z=0$ and fitting only for $b \sigma_{8}\left(z_{p}\right)$.

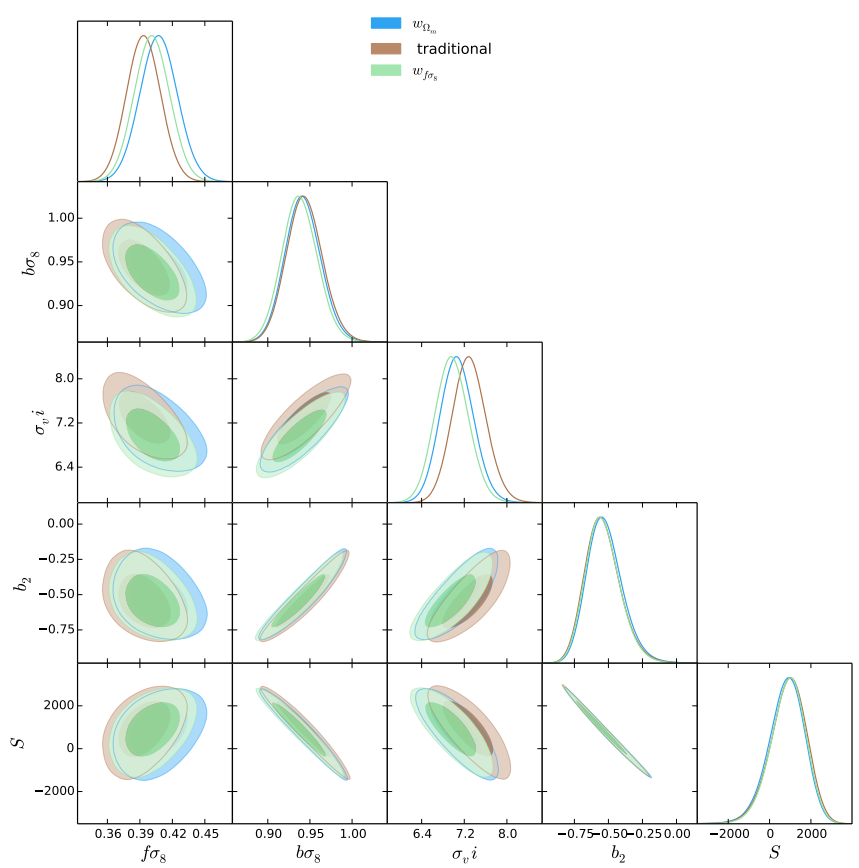

Figure 8. The comparison between the redshift weights analysis and the traditional analysis. Likelihood contours for $f \sigma_{8}, b \sigma_{8}, \sigma_{v}$ $b_{2}, S$ quantities, at their pivot redshift values. Blue likelihood contours show the results obtained with the $\Omega_{m}\left(q_{i}\right)$ analysis; green contours show the results from the $f \sigma_{8}\left(p_{i}\right)$ analysis. Brown contours indicate the results obtained with the traditional analyisis.

\section{CONCLUSION}

In the current work we present a new approach to measure redshift space distortions when dealing with surveys covering a wide redshift range; the redshift weights, applied to each galaxy within the sample, act as a smooth window on the data, allowing us to compress the information in the redshift direction without loss of information. In this analysis we applied the redshift weighting technique to investigate small deviation from the $\Lambda \mathrm{CDM}$ framework; we selected two different parametrization, allowing for deviation in the matter energy density and the growth rate evolution. We derived multiple sets of weights to optimize each order of those deviations. We extended the window function derivation in order to account for the redshift evolution of the power spectrum.

We compared the results obtained for the different parameters with the traditional analysis, i.e. the analysis perfomed considering the clustering as constant in the whole volume. We found that the redshift weights technique gives unbiased constraints for the whole redshift range, in full agreement with the traditional analysis performed at the effective redshift.

The constraints obtained fully validate the analysis (Ruggeri et al. 2017 in prep) to measure RSD on the eBOSS quasars sample where the error expected on $f \sigma_{8}$ is about $5 \%$. To apply the same pipeline to future surveys aiming at $\%$ level accuracy further work will be required; firstly we will need to consider quadratic deviations in the evolution for both the $q_{i}$ and the galaxy bias parameters. In this work we only accounted for those deviations to test the robustness of the fits whereas the signal expected from the quasars sample will not be able to constrain the quadratic evolution.

Another important and interesting aspect would be to account for the AP parameters and their evolution in redshift. To perform such analysis, a set of $N$-body simulations that accurately describe non linearities/light-cone evolution is also required, to reduce the degeneracies and provide a lower statistical error. We here only considered the growth alone, with better data we would be able to include both $\mathrm{AP}$ and growth. For the eBOSS sample, the constraints are too weak to consider this.

\section{ACKNOWLEDGEMENTS}

RR thanks Dr. VP, Dr. RR and Dr. GP for all the support provided. RR and WJP acknowledges support from the European Research Council through the Darksurvey grant 614030, from the UK Science and Technology Facilities Council grant ST/N000668/1, WJP acknowledges the UK Space Agency grant ST/N00180X/1.

Funding for SDSS-III and SDSS-IV has been provided by the Alfred P. Sloan Foundation and Participating Institutions. Additional funding for SDSS-III comes from the National Science Foundation and the U.S. Department of Energy Office of Science. Further information about both projects is available at www.sdss. org. SDSS is managed by the Astrophysical Research Consortium for the Participating Institutions in both collaborations. In SDSS- III these include the University of Arizona, the Brazilian Participation Group, Brookhaven National Laboratory, Carnegie Mellon University, University of Florida, the French Participation 
Group, the German Participation Group, Harvard University, the Instituto de Astrofisica de Canarias, the Michigan State / Notre Dame / JINA Participation Group, Johns Hopkins University, Lawrence Berkeley National Laboratory, Max Planck Institute for Astrophysics, Max Planck Institute for Extraterrestrial Physics, New Mexico State University, New York University, Ohio State University, Pennsylvania State University, University of Portsmouth, Princeton University, the Spanish Participation Group, University of Tokyo, University of Utah, Vanderbilt University, University of Virginia, University of Washington, and Yale University. The Participating Institutions in SDSS-IV are Carnegie Mellon University, Colorado University, Boulder, Harvard- Smithsonian Center for Astrophysics Participation Group, Johns Hopkins University, Kavli Institute for the Physics and Mathematics of the Universe Max-PlanckInstitut fuer Astrophysik (MPA Garching), Max-PlanckInstitut fuer Extraterrestrische Physik (MPE), Max-PlanckInstitut fuer Astronomie (MPIA Heidelberg), National Astronomical Observatories of China, New Mexico State University, New York University, The Ohio State University, Penn State University, Shanghai Astronomical Observatory, United Kingdom Participation Group, University of Portsmouth, Univer- sity of Utah, University of Wisconsin, and Yale University. This work made use of the facilities and staff of the UK Sciama High Performance Computing cluster supported by the ICG, SEPNet and the University of Portsmouth. This research used resources of the National Energy Research Scientific Computing Center, a DOE Office of Science User Facility supported by the Office of Science of the U.S. Department of Energy under Contract No. DEAC02-05CH11231.

\section{REFERENCES}

Alam S. et al., 2016, ArXiv e-prints

Alcock C., Paczynski B., 1979, Nature, 281, 358

Anderson L. et al., 2014, MNRAS, 441, 24

Ata M. et al., 2017, ArXiv e-prints

Baldauf T., Seljak U., Desjacques V., McDonald P., 2012, Phys. Rev. D, 86, 083540

Beutler F. et al., 2017, MNRAS, 466, 2242

Bianchi D., Gil-Marín H., Ruggeri R., Percival W. J., 2015, MNRAS, 453, L11

Blanton M. R. et al., 2017, AJ, 154, 28

Chan K. C., Scoccimarro R., Sheth R. K., 2012, Phys. Rev. D, 85, 083509

Chuang C.-H., Kitaura F.-S., Prada F., Zhao C., Yepes G., 2015, MNRAS, 446, 2621

Dawson K. S. et al., 2016, AJ, 151, 44

Feldman H. A., Kaiser N., Peacock J. A., 1994, ApJ, 426, 23

Gelman A., Rubin D., 1992, Statistical Science, 7, 457, http://www.stat.columbia.edu/〜gelman/research/ published/itsim.pdf

Hartlap J., Simon P., Schneider P., 2007, A\& A, 464, 399

Kaiser N., 1987, MNRAS, 227, 1

Klypin A., Yepes G., Gottlöber S., Prada F., Heß S., 2016, MNRAS, 457, 4340

Laurent P. et al., 2017, ArXiv e-prints

Lewis A., Bridle S., 2002, Phys. Rev., D66, 103511
Mueller E.-M., Percival W. J., Ruggeri R., 2017, ArXiv e-prints

Planck Collaboration et al., 2016, A\& A, 594, A13

Ruggeri R., 2017

Ruggeri R., Percival W. J., Gil-Marín H., Zhu F., Zhao G.-B., Wang Y., 2017, MNRAS, 464, 2698

Scoccimarro R., 2004, Phys. Rev. D, 70, 083007

Scoccimarro R., 2015, ArXiv e-prints 1506.02729

Song Y.-S., Percival W. J., 2009, J. Cosmology Astropart. Phys., 10, 004

Springel V., 2005, MNRAS, 364, 1105

Taruya A., Nishimichi T., Saito S., 2010, Phys. Rev. D, 82, 063522

Wilson M. J., Peacock J. A., Taylor A. N., de la Torre S., 2017, MNRAS, 464, 3121

Yamamoto K., Nakamichi M., Kamino A., Bassett B. A., Nishioka H., 2006, PASJ, 58, 93

Zhao G.-B. et al., 2016, MNRAS, 457, 2377

Zhu F., Padmanabhan N., White M., 2015, MNRAS, 451, 236

Zhu F., Padmanabhan N., White M., Ross A. J., Zhao G., 2016, MNRAS, 461, 2867 